\title{
Adhesion G Protein-Coupled Receptor F1
}

National Cancer Institute

\section{Source}

National Cancer Institute. Adhesion G Protein-Coupled Receptor F1. NCI Thesaurus. Code C113545.

Adhesion G-protein coupled receptor F1 (910 aa, 101 kDa) is encoded by the human ADGRF1 gene. This protein is involved in G protein-coupled receptor signaling, which may be dependent on the removal of its extracellular domain. 